\title{
Facilitating quality improvement in primary health care by practice visiting
}

\author{
G Eliasson, L Berg, P Carlsson, K Lindström, C Bengtsson
}

\section{Introduction}

During the past decade, demands for effective quality assessment in primary health care have increased considerably. "The third revolution of medical care" has seen a move towards more rigorous assessment of clinical interventions and increasing calls for more accountability. ${ }^{1}$ Although most agree that quality improvement is important there are many views about the best approach to quality assessment. In the United Kingdom and elsewhere medical audit has been a notable component of quality improvement programmes. Its aims have been defined: "Its purpose is to identify opportunities and implement improvements in the quality of medical care; medical training and continuing medical education; and effective use of resources". ${ }^{2}$ This statement makes clear that medical audit can contribute to improving health care and professional development. But audit may have other functions. It can be a tool for securing accountability and could be used to control the performance of general practitioners.

Broadly, there are two approaches to quality assessment: one a process of internal reflection, the other external inspection. Shaw describes a continuum between clinical audit as a voluntary, educational, internal process for which there are no sanctions and external inspection that is more regulatory and may involve sanctions. ${ }^{3}$ Similarly, Sheldon has written of the two poles of audit, one external, done by visitors not personally involved in the work of the unit, and the other internal, carried out by doctors and colleagues, assessing their own work. ${ }^{4}$ Marinker states that "the more external the audit becomes, the more others are drawn into making judgements and the more threatening it is to those who are judged". ${ }^{5}$ In Sweden, where primary health care to a listed or a geographically defined population is usually delivered by well functioning multiprofessional teams (box 1), clinical audit that involves the whole team including nurses and paramedics has more relevance than medical audit that is about the work of physicians. ${ }^{6}$

Monitoring performance-that is, controlling performance against agreed standards-is important and is widely accepted as necessary because of the share in the overall decrease in resources allocated to health care that is being borne by primary health care. ${ }^{7}$ Although there are many stakeholders concerned with improving the quality of care, in our view, it is professional carers who should be driving these initiatives and deciding the best approach. ${ }^{8}$ The lack of clear definition allows for interpretation and adoption to find the best approach to suit different localities. Even medical audit may be done in many different ways-one English survey of the literature identified nine major areas of audit activity (table 1). ${ }^{9}$

PRACTICE VISITING

Practice visiting is a method of assessing various aspects of general practice. Briefly, it includes inspection, observation, analysis, and recording of practice activity followed by feedback of results. The aim is, through understanding problems uncovered by visiting a practice, to promote improvement. ${ }^{10}$ Many accreditation programmes make use of practice visiting. In Canada a programme designed to meet requirements for the certification examination is now used for recertification of general practitioners (GPs). ${ }^{11}$ The Canadian programme is built on analysis of use of resources and assessment by criteria statements of skills and performance of the doctor for common diagnoses. It uses a "chart abstraction" technique, that involves assessing patient records against defined standards of care based on diagnoses and includes taking patients' views into account.

In the United Kingdom, assessment of quality in general practice is well established in selection of trainers. ${ }^{12}$ Criteria have been developed that emphasise the importance of the visiting team. ${ }^{13}$ The United Kingdom model of visiting potential training practices assesses both the trainer and the practice, but focuses on the suitability of the practice for education and training. A document "What sort of doctor?" that assesses the attributes of a doctor's qualifications is well known among GPs in the United Kingdom. ${ }^{14}$ The Royal College of General Practitioners in the United Kingdom have developed an approach to the awarding of a fellowship that is based on the visiting of practices by professional assessors, and focuses on the individual GP analysing video recorded material and interviews. ${ }^{15}$ Assessment of consultation skills and results from interviews are essential, but information about availability, continuity of care, and other characteristics of the practice are also used in

Table 1 Nine major areas of medical audit (after Hughes and Humphrey')

\begin{tabular}{ll}
\hline 1 & Practice activity analysis \\
2 & Case analysis \\
3 & Disease and process audit \\
4 & Seeking patients' views \\
5 & Service indicators and the use of routinely available \\
& information \\
6 & Working in peer groups \\
7 & Practice visiting \\
8 & Practice annual reports \\
9 & Facilitation \\
\hline
\end{tabular}

Accepted for publication 20 January 1998 
- The mean rate at which patients consult general practitioners (GPs) is once or twice a year, visits at home are not common

- Many GPs are members of small care teams in which the district nurse plays an important part

- The clinical GP in Sweden is responsible on average for 2400 people

- Most GPs work in group practices, are responsible for defined geographical areas, and are employed by the County Councils, but there is also, to a lesser extent, private health care

- To consult a Swedish GP costs about $£ 10$, visits to nurses are mostly free

Box 1 Swedish primary health care.

the assessment. The Royal Australian College of General Practitioners has defined a set of minimum quality standards required for practice activities with interview, observation, and assessment ${ }^{16}$ This programme assesses the practice, not the individual GP. In Sweden voluntary inspections of training practices have been used to some extent since 1992 and are under development. ${ }^{17}$

Collecting data that describe the activities of a practice is indispensable for quality improvement exercises. This type of data may be collected for many reasons and claims that it describes the quality of care should always be interpreted with caution. The context and relevance of received data must be discussed and the quality of care should only be assessed within established protocols or guidelines. To be useful such guidelines should have been through a process of local adaptation agreed by GPs and be flexible. ${ }^{18}$ National guidelines can be found in The Netherlands, ${ }^{19}$ but in many countries they are still wanted or under development.

Visits to practices enable judgement of many aspects of health, and according to Irvine: "experienced visiting practitioners can and do make judgements about those aspects of general practice which cannot be quantified or measured numerically". ${ }^{8}$ Observations from mutual practice visits can add value to the work of peer review groups, ${ }^{20}$ but it is important to understand that what happens in a practice visit will be influenced by its perceived purpose-that is, whether participants consider it to be voluntary, educational, or official. $^{21}$

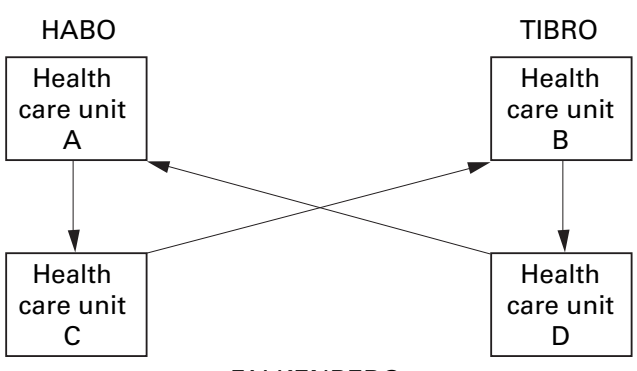

FALKENBERG

Figure 1 Inspection scheme.
The tool box is a portable box with a handle on top, $10 \times 14 \times 8$ inches in size, containing eight shelves with instructions and tools that are updated regularly.

Contents:

How to make an audit programme

Assessment of equipment and premises

Audit of medical quality indicators

(asthma, hypertonia, diabetes, earache,

lower tract urinary infection)

Audit of service quality indicators

Guide Médècine ("incognito" inspection

of a healthcare unit)

Making a patient questionnaire

Practice visiting

Quality circles

Critical incident analysis

Flow chart analysis of referral notes

Empty places (used for documents of ongoing local audit projects)

Box 2 The Swedish quality tool box.

PRACTICE VISITING: A MODEL FOR QUALITY IMPROVEMENT?

Quality improvement work is the responsibility of all members of the care team. Stimulating an interest in quality improvement and a willingness to spend enough time on this aspect of work is crucial if quality improvement is to be accepted and performed with genuine motivation. Ownership of the process by participants is important and the methods used must be obviously feasible, attractive, and easily understandable, but not hierarchical or unduly complex ones. The model of practice visiting could fulfil these criteria and help overcome reluctance of many healthcare centres to be involved in the demanding quality improvement work.

Preparations for a practice visit are central to the whole exercise and include collecting information and measuring performance of practice activities. In Sweden quality improvement methods are available in the Swedish quality tool box (box 2). The tool box was introduced in 1993 by the Swedish Association of General Practitioners $^{22}$ and is designed to assess quality in primary healthcare regardless of size, practice organisation, and management of the unit. From answers to a postal questionnaire received from 799 of 1033 healthcare units in 1996 , most $(89 \%)$ units knew about the existence of the tool box and over half had purchased it. Thus the tool box is available to most primary healthcare centres in Sweden (Lindström K, personal communication 1997). Over $70 \%$ of the units who had purchased it had used at least one of the methods-mostly questionnaires, medical quality indicators, and audit protocols. Lack of time and unavailability of suitable methods for quality assessment were considered by respondents to be the most important obstacles to setting up systems for quality improvement. In the United Kingdom communication within the practice seems to be one important factor among GPs for not participating in audit. ${ }^{23}$ Lack of time, lack of education, and lack of earlier experience of audit are other reasons. ${ }^{24}$ 
- Accessibility by Telephone

- Waiting times in the waiting area

- Staff attitudes

- Continuity of care

- Trust in the GP

- Satisfaction with information

- Overall satisfaction

Box 3 Subjects included in patients' questionnaires.

The idea of developing a method for practice visiting that would focus on facilitating quality improvement and use the tool box as the basis for preparation for the visit came from two GPs working in the primary health centre at Falkenberg. Two GPs at two other health centres interested in this project were identified through a network search-thus four primary health care units in the south western part of Sweden - two in Falkenberg, one in Habo and one in Tibro-that each employed two GPs were involved in this programme. The authors were four of the GPs and an academic member from the department of general practice at a university.

The difference between the use of practice visiting to stimulate quality improvement and its use for accreditation is that to stimulate quality improvement there is a focus on internal communication and implicit variables that aim at improving the quality of care from within, whereas practice visiting for accreditation must always be measurable, objective, and seen to be fair, and is used to define an externally set minimum pass level or standard.

The aims of this paper are to share our experiences of practice visiting as an approach to quality improvement. We describe the preparations for a visit, the process of data collection, the visiting procedures, the approach we took to presentation of observations, assessment of quality of care through a comparison with the assessor's view of the quality in his or her own centre, and the feedback to inspected centres.

\section{Preparation for the programme}

The first stage of planning the practice visits was done by the GPs working from their own centres. This is an important part of the proc-

Table 2 Data collected

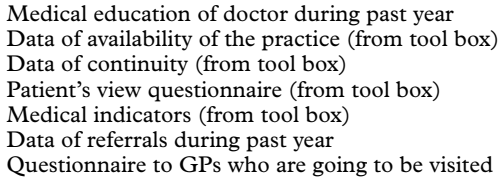

Medical education of doctor during past year

Data of availability of the practice (from tool box)

Data of continuity (from tool box)

Patient's view questionnaire (from tool box)

Medical indicators (from tool box)

Data of referrals during past year

Questionnaire to GPs who are going to be visited

2 Includes questionnaires about availability of doctor and other staff members by telephone, opening hours, and home visit possibilities. "Occupied telephone" problems are assessed by making 20 calls at random.

3 Continuity is measured by studying a randomised sample of 100 patients who have attended the surgery at least three times during one year. Patients who have met different doctors every time, the same doctor at two and at all three visits are counted. 4 The questionnaire includes patient's views on continuity, availability, waiting time, treatment by the doctor and other staff members, confidence, and overall satisfaction.

5 Includes criteria regarding hypertension, asthma, diabetes, earache, and lower tract urinary infections.

7 Includes questions about time on duty, other commitments, subjective valuing of equipment, premises, organisation and management, and workload. ess, and takes place when the objectives of the practice visit can be discussed and agreed by all those involved, when decisions abut the time that is to be invested in the process can be made, the opinions of all the staff about the proposed visits can be sought, and how to use the results that will emerge can be considered. Also the visits procedures, questionnaires, and protocols have to be uniform to allow comparisons with other practices. These are compiled during the planning phase.

Structured protocols for the visits were constructed with four of the nine major areas of audit (table 1), (a) practice visiting, (b) process audit, (c) patient's views, and (d) service indicators. The GPs then met in three half day plenary sessions to discuss, elaborate, and finalise the structure of protocols. The tool box was central to these discussions and methods for quality assessment were selected from it. At these sessions the GPs also agreed other details; wrote vignettes of cases; defined information to be collected, and agreed a visiting scheme (fig 1). There was little difficulty in reaching consensus about targets, procedures, or details of the visiting procedures.

\section{Data collection in preparation for the visit}

The data collection programme involved the preparation and collation of a wide range of details about the practice and the practice activity (table 2) and contributed to describing the elements of quality (table 3). Information was derived for the visits by various methods and from several sources. Explicit and validated quality assessment methods were taken from the tool box. These included, for example, medical indicators for asthma, hypertension and diabetes, and patient questionnaires (box 3). Patient questionnaires were distributed by nurses or receptionists to all patients attending the practice during one week before the visit. These patient questionnaires may be used to assess continuity of care, availability of the practitioners, and attitudes of staff, as well as methods for assessing continuity and availability of care. To save time and unnecessary duplication we agreed that similar patient questionnaires used recently were acceptable. The GPs also completed questionnaires about their continuing medical education in the past year-seminars, conferences, and peer groups attended and books and papers read. Other information such as details of referrals was collected from routine sources within the practice. All this and other relevant information about the unit including demographic data, brief summaries of the health policies of the unit, and patient information were made available to the visiting GPs. The data collection and preparation were done by two GPs from the centre to be visited over a period of two months leading up to the visit. The preparation time was in total 32 hours.

Criteria for the care of common conditions such as asthma, diabetes, hypertension, acute earache, and lower tract urinary infections are contained within the medical indicator section of the tool box and include measures of struc- 
Table 3 Protocol with the visual analogue scale used in practice observation

\begin{tabular}{|c|c|c|c|c|}
\hline & Element & Worse & Equal & Better \\
\hline 1 & Premises & & & \\
\hline 2 & Equipment & & & \\
\hline 3 & Staff resources & & & \\
\hline 4 & Education & & & \\
\hline 5 & Availability & & & \\
\hline 6 & Continuity & & & \\
\hline 7 & Attitudes $v$ patients & & & \\
\hline 8 & Documentation & & & \\
\hline 9 & Routines & & & \\
\hline 10 & Medical quality and security & & & \\
\hline 11 & Cooperation & & & \\
\hline 12 & Preventive measures & & & \\
\hline 13 & $\begin{array}{l}\text { Quality improvement } \\
\text { activities }\end{array}$ & & & \\
\hline
\end{tabular}

ture, process, and outcome. For example, for diabetes care important structural indicators include patient registers, guidelines, and material for patient education. Process criteria include the frequency of audit of the care of diabetic patients (done by examination of patient records), and include records of patients' smoking habits, records of foot care, the proportion of patients whose eyes were examined by an ophthalmologist, and the proportion for whom HbAlc has been measured within the past year. Outcome measures include the proportion of patients with $\mathrm{HbA} 1 \mathrm{c}$ within 1 and 2 SD of normal and the proportion of patients who still smoke tobacco.

Process of assessment by the visiting GP

The list of 13 elements of quality was decided during the preparation phase (table 3 ). This was used by the visitors who for each element judged the quality relative to their own practice. The task of examining appointment schedules, patients' records, and discussing cases was not difficult but the visitors took care to exercise sensitivity, respect, and discretion. The visiting GPs had the option of including interviews or questionnaires to highlight other concerns made obvious by the visiting process after this was completed. The visitors used all the information that had been collected to write a report about the quality of care in the practice. To do this they used the protocol outlined in table 3. Each report took about eight hours to prepare. The visitors used their reports of the assessment of the quality of care during their discussions with the practice at a formal visit. Each visit took about six hours and ended with a short summary of the day, presented to staff members by the visiting GP.

ASSESSMENT OF THE QUALITY OF CARE

With the information collected before the visit and his or her observations and interviews with practice staff the visiting GP compared the quality of care in the practice under review with his or her own practice with a visual analogue scale where better and worse are end points and the distances from the middle (equal) are expressions of quality of care relative to the centre of the visiting GP. Figure 2 shows an example of a completed protocol indicating the assessment of quality made by the visiting GP for all 13 elements assessed. Small deviations were made by some to note unique and more complex findings-for example, the psychological atmosphere of the practice.

We were concerned about the objectivity of this normative approach to assessing aspects of the quality of care. So, to estimate the appropriateness of the visual analogue scale, we estimated the median level attributed to each of the 13 elements for the four practices relative to the equal line on the linear scale. As the assessments were made in a circular way - that is, all GPs were at some time either being assessed or being an assessor - these median values would be expected to be close to the equal line if all GPs were able to be more or less objective in their normative assessments. In fact the calculated median score of the four units for each of the 13 elements of quality (table 3) were all fairly close to zero (fig 3) suggesting that the visitors were handling the scoring in similar ways. Most aspects of the quality of care could be expressed on a linear scale. However, we did not exclude the use of more intuitive comments and other unmeasurable impressions gained by the visitors.

\section{Formal feedback}

Feedback to members of the practice took place about six weeks after the visit. These sessions were led by the GP visitor with the same

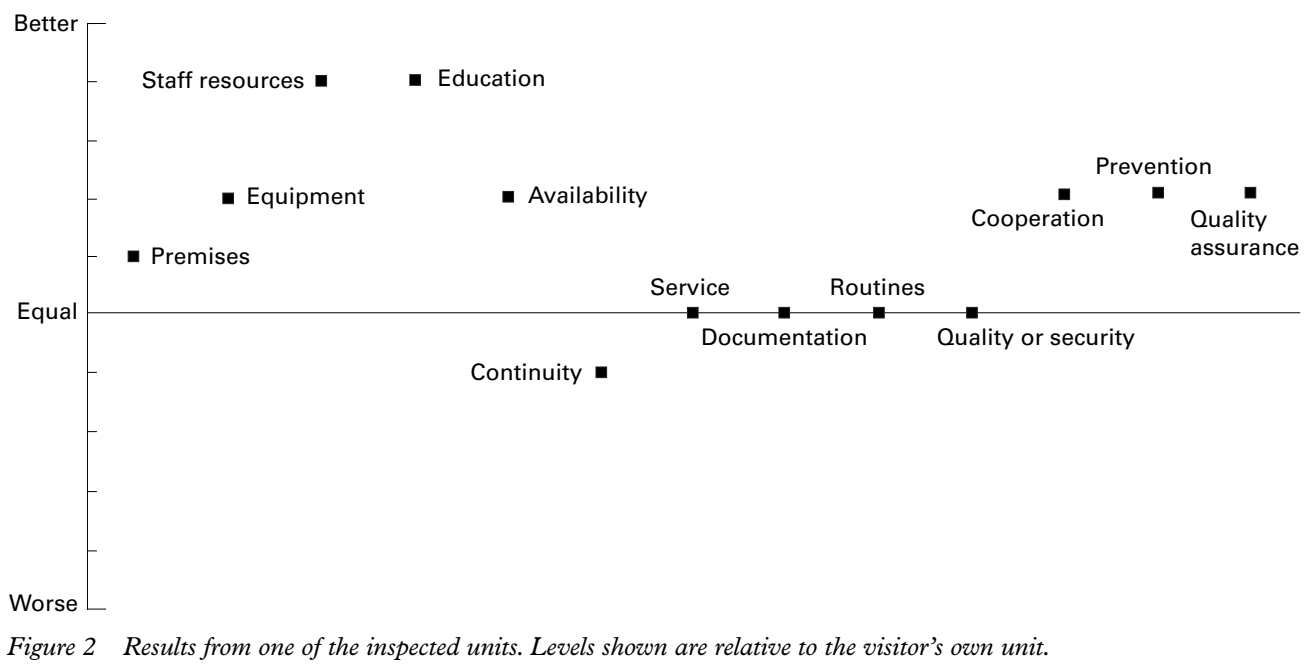


- Briefly clarify matters of fact

- The person in question goes first

- Good points first

- Recommendations - not criticisms

Box 4 Main principles for video consultations, from Pendelton et al. ${ }^{25}$

principles used for video consultations described by Pendleton et al (box 4). ${ }^{25}$ In plenary sessions visiting GPs presented their assessment of strengths and weaknesses of the unit to staff who were given an opportunity to comment on both the information presented and the scoring. The results on the visual analogue scale comparing the practice with the visitor's practice were presented and discussed. Also explicit criteria for the care of people with certain conditions - mainly chronic diseaseswere outlined (box 5). The visitors might also describe their impressions of the practice (box 6 ). Finally, the visitors took part in a discussion with the practice on how to use the assessment to improve the quality of care. At this stage the assessor took on the role of a tutor. In one of the feedback sessions the written report was presented and discussed but in the others the visitors' reports were presented orally.

Discussions about strengths and weaknesses of the inspected unit were easy to start among GPs and their colleagues. Some examples of problems raised through the visit included clear evidence of the poor availability of telephone advice - a problem that the GPs in that practice were unaware of before the visit but which was resolved soon afterwards. In another practice an unacceptable lag was noted between the time of patient consultation and time of completion of administrative tasks by secretaries. This was discussed within the practice and later reported to have been improved by the team. Other problems that were found as the result of the practice visit in a non-computerised practice were the incomplete registration of patients with chronic
- Registration of people with diabetes was not obligatory and many notes lacked record of diagnosis. Agreement that this could be improved by GPs. Target of $100 \%$

- Note about tobacco use in $<50 \%$ of notes. Agreement that this could be improved by GPs. Target of $100 \%$

- $<50 \%$ Patients with diabetes were referred to an eye specialist. Agreement that this could be improved by GPs. Target of $80 \%$

- $94 \%$ Patients had had HbA1c measured in past 12 months. Considered sufficient

- $<50 \%$ Patients had latest HbA1c below or in acceptable range. Should be improved. Discussion followed between GPs and nurses about how to manage this and achieve improvement

Box 5 Comments about diabetes care from visitor and response from practice.

diseases - making it difficult to retrieve records for reports. Computers were introduced into the practice soon after the assessment. In another practice there were recommendations about the fragmented nature of preventive measures being done by many members of the primary healthcare team with little coordination.

\section{Reflection}

The adaptation of practice visiting as a method of quality improvement developed out of a strong need to facilitate quality improvement in primary healthcare centres. This small scale pilot trial has allowed us to gain experience in practice visiting where the main objectives were to make GPs and healthcare teams aware of their strengths and weaknesses and to stimulate them to undertake further quality improvement work. From this we think that practice visiting and use of this type of programme put together in the preparation period can be undertaken by GPs. Feedback reports from these visits involved GPs and other members of

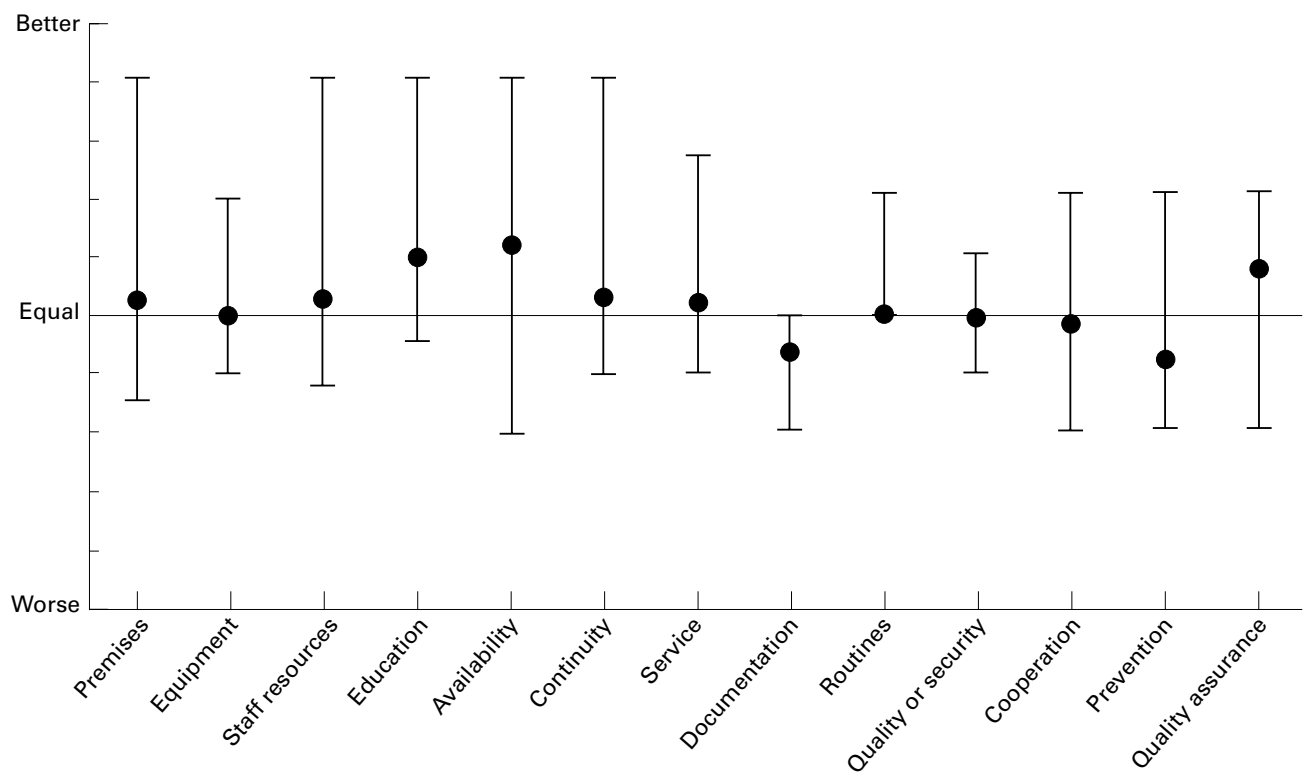

Figure 3 Median, maximum, and minimum levels of quality of the four observed practices. 
- Staff members keen to share problems and to take over work of others when needed

- Atmosphere of the centre open and permitting

- Staff tried to allay anxieties of seriously ill patients

- GPs seem to love their job

- Staff members seemed stressed and in a hurry

- Patient records concise and easy to read and understand

- Both GPs adhere to principles of family medicine and make use of the doctorpatient relation

- Inadequate prioritisation at times when workload is high

Box 6 A visitor's impression of a practice.

the primary care teams in fruitful discussions about the quality of care, and seemed to evoke the will to improve quality and bring about changes.

The health centres that took part in this project were self selected and could all be described as experienced in the field of quality development. We think that colleagues with less experience of quality development but keen to learn and to become engaged in quality improvement in their practices would be willing to adopt the method, because visiting is mostly an attractive and social event.

GPS' VIEWS OF THE PROCESS

GPs had very positive views about the process of being assessed and the responses to feedback sessions were very positive. Six of the eight GPs found that the process had drawn their attention to both known and unknown habits, merits, and shortcomings. One of the GPs gained new insight about quality in the sense that quality should be expressed in terms of performance rather than of medical knowledge. All GPs thought that their investment in time was worthwhile

\section{ASSESSMENT OF THE QUALITY OF CARE}

The tool box was used as a source of explicit criteria for quality improvement but the lack of explicit standards and guidelines in Swedish primary health care is, undoubtedly, a problem. We found that ranking - assessment of the practice's performance compared with the visitor's practice-was an essential component of the feedback discussions. The process lacked explicit standards and perhaps seems arbitrary, but our assessment of the process-as far as it could go with only four participants - suggests that assessment of the quality of care by those visiting GPs was not random.

Another approach to practice visiting would be to employ external, skilled assessors instead of colleagues. This might have improved objectivity, but it was clear that the GPs learnt as much from the process of visiting as from being visited. We also thought that being assessed would be less threatening if confined to a small group of equally experienced colleagues-all involved in the process from the start. Involvement of staff members other than GPs in the visiting process would have provided a broader survey of the practice and added more material to discuss, and would have made the feedback sessions more relevant to all staff members. The feedback session is essential. We noticed the tensed attention of the staff when results were to be delivered by the returning visitor, thus undoubtedly leading to improvements and creating new ideas.

IMPROVING THE QUALITY OF CARE

Evidence of closing the quality circle and being clear that real quality improvement is actually being achieved can only be picked up at a subsequent visit ${ }^{26}$ - and for that sort of assessment explicit criteria will be needed. The links with continued medical education are crucial-as only exceptionally highly motivated GPs will continue quality improvement work in isolation. But if practice visiting is integrated with continued medical education in the form of small group work ${ }^{27}$ the findings and the insights derived from the practice visits, with educational support, can be taken forward and used to promote change.

\section{COMPARISON WITH OTHER SYSTEMS}

Our method of practice visiting differs from the ones established in the United Kingdom, Canada, and Australia in purpose and in approach to information. Our aim was to assess the performance of the practice as a whole and the knowledge and skill of individual doctors were not assessed. This is similar to the Australian scheme that also looks at the whole practice rather than the performance of individual doctors but contrasts with the Canadian scheme and the United Kingdom practice visits for assessment of individual doctors for the fellowship of the Royal College of General Practitioners.

When the main objective of a visit is accreditation, objective scoring is crucial. Skilled trained assessors who work to objective formulated standards are central to the United Kingdom and Canadian schemes and also, but perhaps less so, in the Australian schemes. By contrast, for our purposes, part of the process was that colleagues were assessors and we used comparisons between two subjects. Information from our practice visits- the purpose of which was to facilitate quality improvementshould be the property of the practice that has been visited. Results should only be used for internal discussions and not for anything official. If so, it should be easier for team members to discuss problems and weaknesses that emerge through the visit. Frank internal discussions are central to effective quality improvement. Thus although the practice visits were carried out by an external assessor, the main objective was to encourage internal reflection on aspects of the quality of care within a practice and thus combine both elements of quality assessment. This minimised the threats to those being assessed but provided enough stimuli for real quality improvement to take place. 
If quality improvement is to be integrated further into routine practice it must be attractive, relevant, interesting, nonthreatening, and easy to do. This study indicates that practice visiting can work as a method of quality assessment by self selected GPs. Notwithstanding the time and effort that had to be invested by the participating GPs, the attributes of the scheme make it likely be adopted by professionals. With the tool box as a focus for preparation, some of the barriers to quality improvement work can be overcome. Although this small study was done in Sweden, this approach is likely to be applicable in other countries. Further research and evaluation of how the practice visiting could be used on a larger scale should be used to implement quality improvement work in the most effective way.

1 Relman AS. Assessment and accountability. The third revolution in medical care. $N$ Engl f Med 1988;319:1220-2.

2 Shaw CD. Foint guidelines on assessment of speciality medical audit. London: King's Fund Centre Medical Audit audit. London: Krogramme, 1989.

3 Shaw CD. Aspects of audit. 1. The background. BMF 1980; 280:1256-8.

4 Sheldon MG. Medical audit in general practice. London: Royal College of General Practitioners, 1982. (Occasional paper 20.)

Marinker M. Performance review and professional values. In: Pendleton DA, Schofield TCP, Marinker M, eds. In pursuit of quality. London: Royal College of General Practitioners, 1986

6 Baker R, Presley P. The practice audit plan. A handbook of medical audit for primary health care teams. Bristol: Royal College of General Practitioners, Severn Faculty, 1990.

7 Stone DH. Proposed taxonomy of audit and related activities. F R Coll Physicians Lond 1990;24:30-1.

8 Irvine DH. Standards in general practice: the quality initiative revisited. Br f Gen Pract 1990;40:75-7.

9 Hughes J, Humphrey C. Medical audit in general practice. A practical guide to the literature. London: King's Fund Centre, 1990.
10 Lawrence M, Schofield T. Medical audit in primary health care.Oxford: Oxford University Press, 1993:210-16. (Oxford general practice series 25.)

11 The College of Family Physicians of Canada. Practice assessment program. Mississauga, Ontario, Canada: CFPC.

12 Joint Committee on Postgraduate Training for General Practice. Criteria for the selection of trainers in general practice. London: JCPTGP, 1976.

13 Schofield T, Hasler J. Approval of trainers and training practices in the Oxford region: evaluation. BMF 1984;288: 538-9, 612-4, 688-9.

14 Royal College of General Practitioners. What sort of doctor? Assessing quality of care in general practice.London: Royal College of General Practitioners, 1985. (Report from general practice 23.)

15 Royal College of General Practitioners. Fellowship by assessment. London: Royal College of General Practitioners, 1995. (Occasional Paper 50.)

16 The Royal Australian College of General Practitioners. Entry standards for general practice accreditation. Draft standards for field testing and trials. Rozelle, NSW, Australia: RACGP, 1994.

17 Johnson K. Stiftelse bildad för SPUR-inspektionerna. (Foundation created for SPUR-inspections.) Läkartidningen 1993;90:2102.

18 Grol R. Standards of care or standard care? Scand f Prim Health Care 1993;11(suppl 1):26-31.

19 Grol R. Development of guidelines in general practice. $\mathrm{Br} f$ Gen Pract 1993;43:146-51.

20 Grol R, Lawrence M. Quality improvement by peer review. Oxford: Oxford University Press, 1993:81-8. (Oxford General Practice Series 32.)

21 Irvine DH. Standards in general practice: the quality initiative revisited. Br F Gen Pract 1990;40:75-7.

22 SFAM, Landstingsförbundet, Socialstyrelsen, SPRI. Proj no 804. Verktygsladan. (The tool box.) Stockholm: Landstingsförbundet; 1993.

23 Baker R, Robertson N, Farooqi A. Audit in general practice: factors influencing participation. BMF 1995;311:31-4.

24 Chambers R, Bowyer S, Campbell I. Investigation into the attitudes of general practitioners in Staffordshire to medical audit. Quality in Health Care 1996;5:13-9.

25 Pendleton D, Schofield T, Tate P, et al. The consultation: an approach to learning and teaching. Oxford: Oxford University Press, 1993:68-71. (Oxford General Practice Series 6.)

26 Smith T. Medical audit. Closing the feedback loop is vital. BMF 1990;300:65.

27 Marinker M, Scott M. Small group work. In: Marinker M, ed. Medical audit and general practice. $2 n d$ ed. London: BMJ Publishing Group, 1995:186-96. 